\title{
Analgesic Effects of Transcranial Direct Current Stimulation on Central Neuropathic Pain in Spinal Cord Contusive Rat Model
}

\author{
Kyung-Yoon Kim, Ki-Chol Sim \\ Department of Physical Therapy \\ Dongshin University, Naju, Korea \\ Hyun-Seung Kim \\ Department of Physical Therapy \\ HealingsHospital, Uijeongbu, Korea \\ Wan-Suk Choi, Gi-Do Kim \\ Department of Physical Therapy \\ International University of Korea, Jinju, Korea
}

\begin{abstract}
The aim is to investigate the analgesic effect of transcranial direct current stimulation(tDCS) on central neuropathic pain(CNP) in spinal cord contusive rat model. Twenty Sprague-Dawley rats(250 $\pm 50 \mathrm{~g}$, male) were used. Thoracic spinal cord(T10) was contused using New York University(NYU) spinal cord impactor. The animals were randomly assigned to two groups; Group I: Nontreatment after SCI induction(n=10), Group II: application of tDCS(0.1 mA, 20 min/time, 2 times/day, 5 days/6week) after SCI induction(n=10). Assess the effect of tDCS using the Basso Beattie Bresnahan(BBB) locomotor rating scales, Touch test ${ }^{T M}$ sensory evaluator(TTSE), Plantar test ${ }^{\circledR}$ after contusion at the $2^{\text {nd }}, 3^{\text {rd }}, 4^{\text {th }}, 5^{\text {th }}, 6^{\text {th }}$ week and the immunohistochemistric response of c-fos in the thalamus, cerebral cortex after contusion at the $3^{\text {rd }}, 6^{\text {th }}$ week after SCI. The scores of BBB scales were significantly different from $3^{\text {rd }}$ week. TTSE were different significantly over time, but there were no differences at each evaluation times on between-measure time effects. Plantar test were different significantly over time and there were difference at the $4^{\text {th }}$, $6^{\text {th }}$ week after SCI on between-measure time effects. Also,immunohistochemistric response of c-fos was reduced significantly from $3^{\text {rd }}$, $6^{\text {th }}$ week after SCI in tDCS group compared with control group in thalamus and cortex. These results identified that tDCS of non-invasive therapeutic method may have beneficial analgesic effect on CNP after SCI with behavioral test and immunohistochemical test.
\end{abstract}

Keywords: Contusive Spinal Cord Injury, Central Neuropathic Pain, tDCS, Anagesic Effect, c-fos.

\section{INTRODUCTION}

A central neuropathic pain caused by a spinal cord injury may be divided into a nociceptive pain by over-activity of peripheral sensitization receptor and a neuropathic pain by nerve damage.They act as the limit of several physiotherapies because they cause heavy pains by damage of nerve cells or axons consisting of CNS(central nervous system)[1].The common symptoms of neuropathic pain are allodynia(the pain caused by soft stimulus), hyperalgesia(the pain of bigger intensity induced by the stimulus of the intensity equivalent to induce a normal pain) and a spontaneous pain(the pain caused by without external stimulus)[2]-[4].Recently, several

\footnotetext{
*Corresponding author. E-mail :pray1980@hanmail.net
} Manuscript received Nov. 07, 2011 ; accepted Jan.27, 2012 experimental animal models showing similar activities to allodynia, hyperalgesia and spontaneous pain frequently observed in patients with a spinal cord injury have been developed, and so there have been various tries and challenges to discover the mechanism[5].The clinical patterns of rats for a neuropathic pain, such as shake of the head toward the stimulus site, sharp screaming, complex regional pain syndrome accompanying edema and color change, allodynia, and heat hyperesthesia, were reported[1],[6].However, mechanical allodynia and heat hyperalgesiawere regarded as the most representative presenting symptoms[7],[8].

The neuropathic pain mechanism caused by a spinal cord injury is explained by hyper-excitability of spinal nerve cells and effacement of inhibitory control (disinhibition) from an upper nervous system[9]. Various drugs such as NMDA antagonist, tramadol agent and anti-depressant opioids to treat 
the pain have been used, but the results have not been satisfactory. For non-drug treatment method such as spinal cord stimulation, dorsal root neurectomy and deep cranial stimulation have been tried, but lots of limitations follow [10]. Accordingly, a way to relieve a pain by modulating direct cranial activity for a specific nervous structure has been considered.As a non-invasive brain stimulus of central neurostimulation, a way to stimulate a braineffectively and safely using tDCS(trans-cranial direct current stimulation) has been suggested. The tDCS is a technique that cause a change on excitability of the local part neuron in the brain by making a soft current flow to scalp[11],[12], which has been known to control the displacement potential of neuropil by affecting the activity of sodium ion channel and calcium ion channel of neuropil and thereafter change the activity of thecranialneurogen[11]-[13].The modus operandi has been estimated by the change of plasticity on synaptic connection in accordance with the change on efficiency of the $\operatorname{NMDA}(\mathrm{N}$ methyl-D-aspartic acid) receptor[11, 14].So far, most of the research regarding the tDCS have reported that it affects excitability cognitive function of cerebral cortex, exercise rehabilitation for men and can control them[15],[16].Alsosomeresearch regarding a pain by a peripheral lesion[17] or a central pain after a spinal cord injury have been reported[18]. However,such research estimates just the cause of effect based on clinical observation if the effect of the tDCS is testified.In the meantime, immunohistochemical staining of c-fos protein which is a substance producing an immediate early gene is widely used for to verify the level of pain and analgesic effect[19]. The c-fosproteinis expressed on the site in relation to the pain on spinal cord and brain[20] and makeseffect of analgesic substance possible quantify the as a marker of nerve action and confirm the neuroanatomical location[21].

As part of ways to investigate the variouseffect shown by activating a brain with a non-invasive method, this study tried to prove the effect of the tDCS by observing the level of threshold value of pain behavior(the threshold value of avoidance response), latency assessment and onset of c-fos on thalamus which has a knownneurotransmitterrelated to pain.

\section{MATERIALS AND METHODS}

\subsection{Laboratory animals}

This study used 20 Sprague-Dawley typed rats weighting about $250 \pm 50$ g(8 weeks old, masculine, Korea Laboratory Animal Association) after making spinal cord contusion to them from $2.5 \mathrm{~cm}$ of height of fall, dividing them into two random groups. The temperature of insectorywas kept at $25 \pm 1{ }^{\circ} \mathrm{C}$ and the humidity at $55 \pm 10 \%$. Shading of the insectorywas changed in the cycle of 12 hours and feeding including water was free during the experimental period.

\subsection{Spinal cord contusive rat model}

To manufacture a spinal cord contusive rat model, general anesthesia was applied to rats by anesthetic gas mixing $70 \%$ $\mathrm{N}_{2} \mathrm{O}, 28.5 \% \mathrm{O}_{2}$ and $1.5 \%$ enflurane and then executed depilation according to the spinal cord line. Then, their pleural effusion fluid was disclosed by 10th dorsal spine laminectomy on an operating table. Next, the rats were moved to a spinal cord impactor(NYU, USA).Asupporting bar was fixed so that an impactor was located in the vertical upwards of the dura mater of dorsal spine disclosed. The spinal cord contusive rat model was then induced by removing the fixed supporting bar at the fall height of $2.5 \mathrm{~cm}$ (Fig. 1).

After inducing the spinal cord contusion, the muscles around the spinal cord were sutured first according to each layer of muscle, and the operative wound was disinfected by PovidoneOdide. After dressing, their body temperature was maintained until rats recovered. All the rats were bred under the environment of constant temperature and humidity. To prevent infection, $1 \mathrm{mg} / \mathrm{kg}$ of sulfuric acid Gentamicin ${ }^{\circledR}$ (Korea United) diluted by normal saline solution was injected into their muscles once a day for a total of five days.Also, to prevent dehydration and electrolyte metabolic error, 5 ml of Hartman Solution was injected into their hypoderm once a day.Additionally to control neurogenic vesical dysfunction, voiding was executed by stimulating their abdomen three times per day for seven days until they accomplished voluntary voiding.

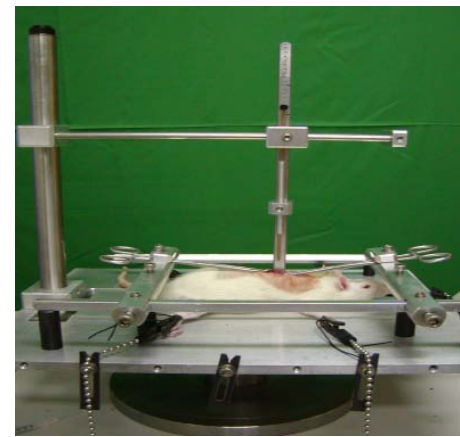

Fig.1. Spinal cord impactor

\subsection{Transcranial direct current stimulation(tDCS)}

The rats with induced spinal cord injury were divided into a non-stimulus group(control group, $\mathrm{n}=10$ ) and the tDCS group (experimental group, $\mathrm{n}=10$ ) randomly, and then same conditions were applied to them except the factor of presence or absence of execution of the tDCS.Forthe tDCS application, a direct current electro stimulator(Cybermedic Co., Korea) that is able to control the intensity in the unit of $0.1 \mathrm{~mA}$ was used in accordance with Kim, Sang-Joon[22]'s method.A positive electrode was fixed and applied to the model of plastic cup in order to attach to a trans-cranial site of cerebral cortex. A negative electrode was applied to the neck in order to block the shunting effect of the electricity(Fig. 2).

To reduce the electric resistance between the skin and electrode, gel was applied to their skin after depilation.The diameter of a positive electrode is $1 \mathrm{~cm}$ and the plastic cup model was filled with the gel.The electric intensity for applying an electric current is $0.1 \mathrm{~mA}$ and the duration of the stimulation was 20 minutes each time. The electric current was constantly applied for six weeks twice a day and five days a week from the fourth day after the spinal cord injury. 


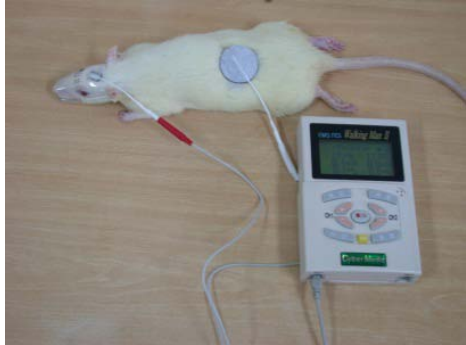

Fig.2. tDCS

\subsection{Behavioral examination for pain}

To examine the analgesic effect of the tDCS on the site below of the site damaged by a spinal cord injury, for both a control group and an experimental group, functional behavioral assessment(FBA) and avoidance response assessment for mechanical allodynia and heat hyperalgesiawere executed before the spinal cord injury and after the injury at an interval of a week from second to eighth week.

2.4.1Functional behavioral assessment(FBA):BBB scale[23] was used to assess the motor function of hind limbs of rats induced spinal cord contusion. The BBB scale departmentalized the assessment factors such as motion of the hind limbs and toes and the degree to control a tail centered motion range and weight support, and then the scale scored them. The motor function was assessed by $13 \times 90 \times 10 \mathrm{~cm}^{3}$ sized rectangle acrylic gait analysis arthrosis.

2.4.2 Threshold value assessment of avoidance response: This study assessed a threshold value of avoidance response using Touch test ${ }^{\mathrm{TM}}$ sensory evaluator(TTSE) for mechanical allodynia caused by a neuropathic pain after inducing a spinal cord contusion. A wire netted acrylic box $\left(20 \times 25 \times 13 \mathrm{~cm}^{3}\right)$ was installed $35 \mathrm{~cm}$ high from the floor and then an inclined mirror was installed under the box in order to observe their behavior response effectively. Mechanical stimulus was applied to third metatarsal site on left hind foot, standing vertically von Frey filament(North Coast Medical Inc., Canada).The threshold value (paw withdrawal mechanical threshold; PWT) of avoidance response against mechanical stimulus was regarded by the minimum bending force avoiding voluntarily legs three times or more among 5 times stimulations using filament (1.0, 1.4, 2.0, 4.0, 6.0, 8.0, 10.0, 15.0 and 26.0g)[24].If the bending force of von Frey filament is excess of $10 \%$ of the weight of the rat, it will be result that the whole leg is passively pushed up.So, the maximum applicable bending force was $26.0 \mathrm{~g}$ in this study[25]. Lifting up sole, licking, shaking off or running away when applying the stimulus were regarded as positive reaction of avoidance response.

2.4.3Latency assessment of avoidance response: This study measured the latency of avoidance response usingthePlantar test ${ }^{\circledR}$ (hot plate analgesia meter, HAVARD Ltd., UK).Itrepeated twice in the unit of 0.1 second at the interval of five minutes after locating infrared-ray heat radiator $\left(50^{\circ} \mathrm{C}\right.$ of heat source and an acrylic panel with $46^{\circ} \mathrm{C}$ of conductive heat) on the sole of hind limb. The latency of avoidance response was decided as the time from applying heat stimulus to occurrence of the avoidance, and the cut-off time of devices was 30 seconds because of tissue injury.

\subsection{Histological examination}

2.5.1Making of tissue slice: This study anesthetized respectively five rats of each group through intraperitoneal injection $(0.6 \mathrm{mg} / \mathrm{kg}$ ) with Rompun(Bayer Korea) at third and sixth week after inducing the spinal cord contusion. Next, this study cleaned heart perfusion with $0.9 \% \mathrm{NaCl}$ and then with $4 \%$ neutral paraformaldehyde( $0.1 \mathrm{M}$ phosphate buffer ph 7.4 ) after removing blood. After prefixation, the brains were extracted and fixed with same fixative for 24 hours at the room temperature. The fixed brain tissue was cut to a thickness of 5 «musing a tissue slicer(microtom; Sakura 2040, Japan). With reference to the article of Paxinos and Watson[26], the abutted tissue in the rear of Bregma(Bregma -1.30, -3.15, -7.64) was selected. Immunohistochemically stained c-fos protein was applied to the brain slice.

2.5.2Immunohistochemical staining method: Immunohistochemical staining is a way to dye the c-fos expressed in a neurogen, which cleaned the brain slice three times at an interval of ten minutes with $0.01 \mathrm{M}$ PBS(phosphate buffer saline) and applied $1 \%$ sodium borohydride for an hour in order to remove the remaining fixative. As for preprocessing ofimmunohistochemical staining, $0.3 \%$ hydrogen peroxide was applied to the slices for 20 minutes. After cleaning them several times with PBS, normal blocking serum was cultivated for 20 minutes using Novostain Super ABC Kit(Novocastra Lab., Benton Lane, UK). After applying anti-c-fos antibody (abcam, 7963 , UK) diluted respectively at the rate of 1:1,000 for 24 hours at $4^{\circ} \mathrm{C}$,clean them with PBS. Then the slices were cultivated for 30 minutes with diluted biotinylated secondary antibody solution. They were cleaned again with the PBS, cultivated by Novostain Super ABC Reagent for 30 minutes, and then cleaned again with the PBS. For the purpose of color development, DAB(Serotec Ltd, BUF021B, UK) was applied to the slice samples for ten minutes and then counterstaining was executed by Mayer's Hematoxyline(Sigma, MHS-32, USA).They were cleaned by running water for five minutes, dried and sealed so that it was observed through a common dehydration process.

2.5.3Histological analysis: This study assessed slice samples at negative(-), mild(+), moderate(++), and severe(+++), dividing them with a semiquantitative manner using Imageproplusver 4.0 for windows(media cybernetics, USA) connecting CCD camera(Foculus, Germany) installed on an optical microscope(Olympus BX50, Japan) to a personal computer after checking thalamus on the brain sample with reference to the article of Paxinos and Watson[26].

\subsection{Statistical method}

For the statistical analysis of this study, SPSS 12.0 ver. for window ${ }^{\circledR}$ was used. The results of all experiments were expressed as a mean and standard deviation.Repeatedmeasuresof ANOVA was used for the 
comparison between acontrolgroup and an experimental group and the comparison on change of pain threshold value in accordance with the lapse of time and independent t-test was used for the comparative verification on each time of measurement. If ' $\mathrm{p}$ ' value is less than 0.05 , statistical significance level was used.

\section{RESULTS}

\subsection{Functional behavioral assessment(FBA)}

The value of the BBB scale for the experimental group was 21points before inducing a spinal cord contusion, but it was $7 \pm 1.24$ points at second week after the spinal cord contusion(one of the control group was $6.1 \pm 1.19$ points) and it was $16.2 \pm 1.54$ points at sixth week after such induction for observation according to follow-up observation.This showed significant rehabilitation of motor function compared with the control group $(\mathrm{p}<0.05)$ and the rehabilitation level of motor function according to time lapsed was meaningful too $(\mathrm{p}<0.05)$. Also, both groups showed meaningful difference since third week after the induction $(\mathrm{p}<0.05)$ (Fig. 3).

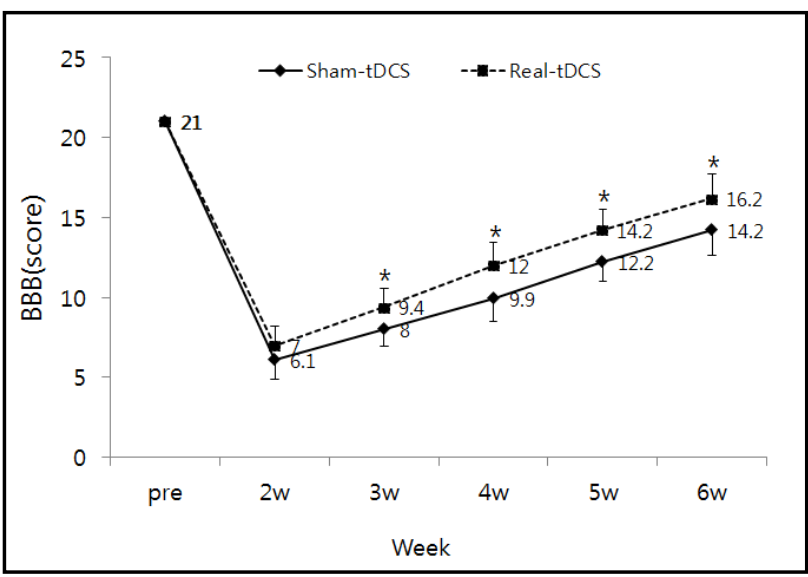

Fig.3. Change on BBB with time

\subsection{Threshold value assessment of avoidance response}

In assessment of a threshold value of avoidance response using the TTSE test, the value of the experimental group before the induction of the spinal cord contusion was $13 \pm 2.58 \mathrm{~g}$ and the one of the control group was $12 \pm 2.58 \mathrm{~g}$. Thus, there was no significant difference observed between the two groups. At second week after the inductionof the spinal cord contusion, the experimental group showed the threshold value of $4.2 \pm 1.47 \mathrm{~g}$ (the value of control group was $3.6 \pm 1.57 \mathrm{~g}$ ), which meant that the threshold value of avoidance response sharply decreased. The threshold value of avoidance response according to the time lapsed showed significant difference between the two groups $(\mathrm{p}<0.05)$, but the value at each time of measurement did not show meaningful difference(Fig. 4).

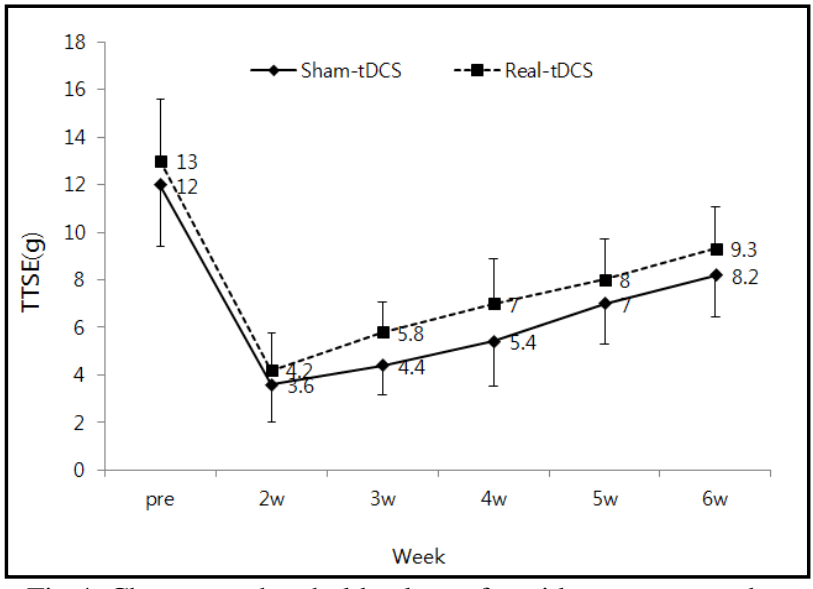

Fig.4. Change on threshold values of avoidance response by time lapsed

\subsection{Latency assessment of avoidance response}

For the latency assessment of avoidance response using the Plantar test ${ }^{\circledR}$, the latencies of both groups were respectively, $27.84 \pm 0.86$ seconds and $28.45 \pm 0.71$ seconds before the inductionof the spinal cord contusion. These did not show any significance. The latency of the control group was 15.01 \pm 3.17 seconds at second week after the induction which showed a clear and obvious latency delay, and since then, the latency delay gradually shortened and the latency of avoidance response according to time lapsed showed significant difference compared with the one of experimental group $(\mathrm{p}<0.05)$. In case of the experimental group, it showed latency delay of $17.97 \pm 3.5$ seconds at second week after the induction. Such latency delay gradually shortened, which was $20.72 \pm 2.25$ seconds at fourth week after the induction (one of control group was $16.41 \pm 3.45$ seconds) and 22.67 \pm 2.78 seconds at sixth week(one of control group was 19.76 \pm 2.49 seconds). So, this study could confirm the significant effect of the tDCS on pain release for heat hyperalgesia after the spinal cord injury because the latency of avoidance response was significantly shortened( $\mathrm{p}<0.05)$ (Fig. 5).

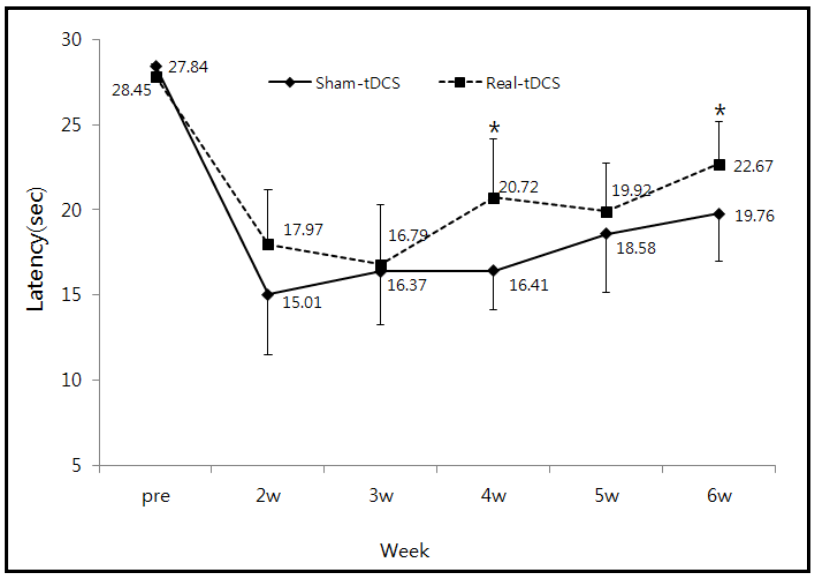

Fig.5. Changes on latency of avoidance response by time lapsed

\subsection{Immunohistochemical reactions of c-fos}


As a result of observation through immunohistochemical staining of c-fos in each brain tissue at third and sixth week after the induction spinal cord injury, to understand analgesic effects of the tDCS, the control group showed strong positive reaction( $(+++)$ at third week and the experimental group a medium positive reaction $(++)$ at third week. At sixth week after the induction the control group showed medium positive reaction(++) and the experimental group a weak positive reaction $(+)$ (Table 1) (Fig. 6). This study confirms that the immune positive reaction of the c-fos of the experimental group was decreased by applying the tDCS.

Table 1. Semi-quantitative assessment of c-fos

\begin{tabular}{|c|c|c|}
\hline \multirow{2}{*}{ Group } & \multicolumn{2}{|c|}{ Assessment Date } \\
& 3rd Week & 6th Week \\
\hline Control Group & +++ & ++ \\
\hline Experimental Group & ++ & + \\
\hline
\end{tabular}

-: negative, +: mild, ++: moderate, +++ : severe
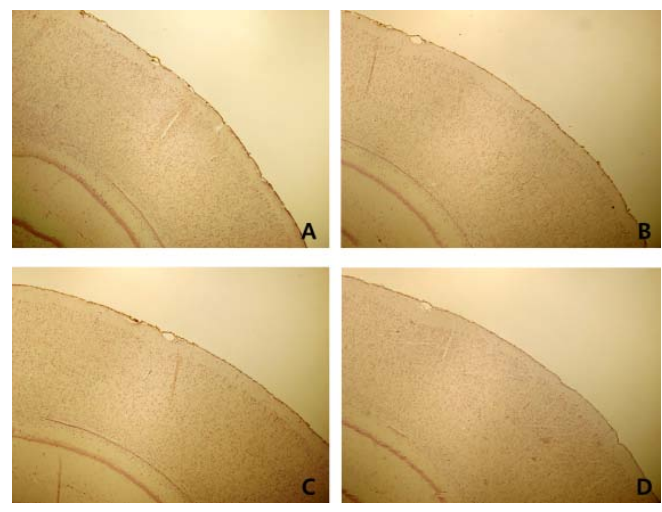

Fig.6.Immunohistochemical reaction of c-fos

A: Control group in $3^{\text {rd }}$ week

B: Experimental group in $3^{\text {rd }}$ week

C: Control group in $6^{\text {th }}$ week

D: Experimental group in $6^{\text {th }}$ week

\section{DISCUSSION}

One of the most difficult and severe pains is various types of central neuropathic pain expressed after cerebral stroke or a traumatic spinal cord injury[27]. In spite of various drug treatments, it is almost impossible to release the pain sufficiently. Accordingly, it needs a new therapeutic approach, and neuromodulation approach like brain stimulation has attracted the concerns from the medical world[18]. Among them, deep brain stimulation shows the result of high possibility[28], but Fregni, et al.[18] reported that the less invasive the type of stimulation, the more effective.

The tDCS, which is one of the non-invasive brain stimulations, improves cheirokinesthesia and promotes implicit motor learning and working memory. When a positive electrode is applied to a primary sensorimotor area andpremotor area, it was reported to be effective inimprovingvisuo-motor learning when applied to an occipital lobe[13],[29] and to improve aphasia or increase the motor function of lower limbs for patients with cerebral stroke[30],[31]. Also, it was reported to be helpful for improving the perception of chronic pain[32],[33] and various symptoms in relation to chronic pain syndrome[18, 34].However, the basic research that is able to explain the mechanism of ethological improvement for such stimulation on a cerebral cortex is very insufficient. So far, several researches for onset patterns of central neuropathic pain in men have been studied[35,36].Whereas, the research ofthelaboratory animals has not been reported or disregarded. So, there were no researches for central pain until now except for motor function[22], blood circulation[37] and anamnesis[38]. Particularly, there is noresearch regarding the cerebral base and histological change in relation to the ethological change about a threshold value using the tDCS.

This study has tried to assess temporal pattern for two main symptoms, mechanical allodynia and heat hyperalgesia, and the change in the c-fos onset on thalamus known as a dolocogenic protein by using the tDCS together with QST(quantitative sensory test) for the onset of central neuropathic pain occurred after the spinal cord contusion in an animal experiment.It utilized the results of the assessment as the basic information and data for the purpose of clinical efficiency of the tDCS against the central neuropathic pain. The established theory for the mechanism of central neuropathic pain had been known.According to a recent report, however, a dafferentiation central pain was caused by the effacement of afferent impulse on the site below of the area damaged in the spinal cord[39,40].A patient misunderstood the stimulus from posterior column of spinal cord as a pain because the inhibitory signal to the dolorific site from a brain stem or thalamus was blocked.Further, the hypothesises that the spinal cord and effaced brain cell creates afferent impulse by emitting action potential voluntarily[39]-[41] waspersuasive.The clinical patterns are thermalgeia, a prick pain, burning sensation, pressure and coldness. In case of men, they depend on subjective appeal.Theyhave various pain patterns and ranges and show complicated patterns in accordance with the severity of spinal cord injury and a mental cause. So, it is very difficult for men to design an effective treatment method[42].On the other hand, a laboratory animal model has a merit to make objective quantification possible because it can assess a threshold value of avoidance response with the TTSE test using the von Frey filament as the QST and the latency of avoidance response due to heat hyperalgesia using the Plantar test ${ }^{\circledR}$.QST protocol was known to be a standard psychophysical test method that is able to assess broad somatosensory perception controlled by the action of a small or big fiber, and simultaneously a way to understand the effacement or noneffacement of various somatosensory pathways [43],[44].Thus, the researcher of this study examined the onset symptom of the central neuropathic pain according to the time lapsed in the viewpoint of two factors - mechanical allodynia and heat hyperalgesia using the TTSE test and Plantar test ${ }^{\circledR}$.

The main results of the QST showed significant difference according to the time lapsed.The threshold values of the avoidance response of both the experimental and the control 
group remarkably decreased at second week after the inductionthe spinal cord contusion in the TTSE and the Plantar test ${ }^{\circledR}$. However, the values gradually increased from third week $(\mathrm{p}<0.05)$. The result of the TTSE test did not statistically show meaningful difference at each time of measurement, but the one of Plantar test ${ }^{\circledR}$ showed significant difference at fourth and sixth week $(\mathrm{p}<0.05)$. Therefore, it was confirmed that the tDCS affects the change of temperature threshold value of the heat hyperalgesiaby A- $\delta$ and $C$ fibers rather than the mechanical allodynia.As a result of using the BBB scale to assess the motor function and recovery, both groups showed meaningful difference from the third week after the induction $(\mathrm{p}<0.05)$. So, this study could confirm that the tDCS affects the motor function as well as the sensory change.

Meanwhile, this study tried to confirm severity of pain and analgesic effects with an immunohistochemical method of c-fos which is an immediate early gene. Bullitt et, al.[45] reported that c-fos significantly increased at a nervous pathway delivering a pain caused by mechanical noxious stimulation or heat noxious heat.Strassman and Vos[46] reported that the feasibility of the c-fos as an index of nervous action against pain stimulus by proving that the onset of c-fos increased in CNS in proportion to the noxious stimulus of peripheral nerve. This study observed the onset level of the c-fos in thalamus and cerebral cortex which is the ascending pain pathway through nociceptive inhibitory action of the tDCS in the upper area of spinal cord. For the experimental group, the positive reaction of the c-fos decreased from third week after the inductionof the spinal cord contusion and showed remarkable decrease at sixth week in both thalamus and a cerebral compared with the control group.

According to all the results above, it was confirmed thatthetDCS has significant analgesic effect for a central neuropathic pain. This study shows that such analgesic effects are to induce the change of electrical characteristics of a neurologic membrane in a stimulus site[47], and inhibit ascending pain transfer of subcortical thalamic nuclei because of the change of local excitabilityandto control abnormal activation of thalamus through cortico-thalamic fibers[48]. This studyconfirms the analgesic effects ofthetDCShistochemically through checking the change of the c-fos protein which is a pain index at thalamus and cerebral cortex. It is necessary for further research and studies of the mechanism to control various factors involved such as the most appropriate intensity, time and site in the effect of tDCSon pain control.

\section{CONCLUSION}

This study confirmed that the heat hyperalgesia showed meaningful difference between the two groups for the two main symptoms, mechanical allodynia and heat hyperalgesia, in follow-up observation since constant applicationof the tDCS for about six weeks using a central neuropathic spinal cord contusive rat model.The change of the c-fos protein confirmedthat meaningful analgesic effect in thalamus and cerebral cortex. Hereby, this study can be the basic foundation of clinical application of the tDCS which has been suggested as the non-invasive method to treat a neuropathic pain.

\section{REFERENCES}

[1] G. Davidoff, E. Roth, M. Guarracinin, J. Sliwa, and G. Yarkony, "Function-Limiting DysestheticPainSyndrome AmongTraumaticSpinal Cord Injury Patients: ACrossSectional Study," Pain, vol.29, no.1, 1987, pp.39-48.

[2] S.B. McMahon, "Mechanism of Sympathetic Pain," Br Med Bull, vol.47, no.3, 1991, pp.584-600.

[3] J. Sato, and E.R. Perl, "Adrenergic Excitation of Cutaneous Pain Receptors Induced by Peripheral Nerve Injury," Science, vol.251, no.5001, 1991, pp.1608-1610.

[4] Z. Seltzer, and Y. Shir, "Sympathetically-Maintained CausalgiformDisorders in a Model for Neuropathic Pain: AReview," J Basic ClinPhysiolPharmacol, vol.2, no.1-2, 1991, pp.17-61.

[5] Y.S. Gwak, "The Mechanism of Central Pain Induced by Spinal Cord Injury," Doctoral Dissertation, TheGraduate School of Yonsei University, 2001.

[6] V. Chapman, J. Ng, and A.H. Dickenson, "A Novel Spinal Action of Mexiletine in Spinal Somatosensory Transmission of Nerve Injured Rats," Pain, vol.77, no.3, 1998, pp.289-296.

[7] P. Siddall, C.L. $\mathrm{Xu}$, and M. Cousins, "AllodyniaFollowingTraumatic Spinal Cord Injury in the Rat," Neuroreport, vol.6, no.9, 1995, pp.1241-1244.

[8] R.P. Yezierski, "Pain Following Spinal Cord Injury: Pathophysiology and Central Mechanisms," Prog Brain Res, vol.129, 2000, pp.429-449.

[9] T.N. Bryce, and K.T. Ragnarsson, "Pain AfterSpinal Cord Injury," Phys Med RehabilClin N Am, vol.11, no.1, 2000, pp.157-168.

[10] Y.K. Bae, S.J. Kim, J.M. Seo, Y.W. Cho, S.H. Ahn, I.S. Kang, H.W. Park, and S.J Hwang, "Effects of Continuous Repetitive TranscranialMagnetic Stimulation on Pain Response in Spinal Cord Injured Rat," Korean Academy of Rehabilitation Medicine, vol. 34, no. 3, 2010, pp. 259264.

[11] D. Liebetanz, M.A.Nitsche, F. Tergau, and W. Paulus, "Pharmacological Approach to the Mechanisms of Transcranial DC-Stimulation-Induced After-Effects of Human Motor Cortex Excitability," Brain, vol.125, no.10, 2002, pp.2238-2247.

[12] M.A. Nitsche, and W. Paulus, "Excitability Changes Induced in the Human Motor Cortex by Weak TranscranialDirectCurrent Stimulation," J Physiol, vol.527, no.3, 2000, pp.633-639.

[13] M.A. Nitsche, A. Schauenburg, N. Lang, D. Liebetanz, C. Exner, W. Paulus, and F. Tergau, "Facilitation of Implicit Motor Learning by Weak TranscranialDirectCurrent Stimulation of the Primary Motor Cortex in the Human," J CognNeurosci, vol.15, no.4, 2003, pp.619-626.

[14] M.A. Nitsche, K. Fricke, U. Henschke, A. Schlitterlau, D. Liebetanz, N. Lang, S. Henning, F. Tergau, and W. Paulus, "Pharmacological Modulation of Cortical Excitability Shifts Induced by TranscranialDirectCurrent 
Stimulation in Humans," J Physiol, vol.553, no.1, 2003, pp.293-301.

[15] A. Antal, D. Terney, C. Poreisz, and W. Paulus, "Towards UnravellingTask-Related Modulations of NeuroplasticChangesInduced in the Human Motor Cortex," Eur J Neurosci, vol.26, no.9, 2007, pp.26872691.

[16] B.R. Webster, P.A. Celnik, and L.G. Cohen, "Noninvasive Brain Stimulation in Stroke Rehabilitation," NeuroRx, vol.3, no.4, 2006, pp.474-481.

[17] P.S. Boggio, E.J. Amancio, C.F. Correa, S. Cecilio, C. Valasek, Z. Bajwa, S.D. Freedman, A. Pascual-Leone, D.J. Edwards, and F. Fregni, "Transcranial DC Stimulation Coupled with TENS for the Treatment of Chronic Pain: APreliminaryStudy," Clin J Pain, vol.25, no.8, 2009, pp.691-695.

[18] F. Fregni, P.S. Boggio, M.C. Lima, M.J. Ferreira, T. Wagner, S.P. Rigonatti, A.W. Castro, D.R. Souza, M. Riberto, S.D. Freedman, M.A. Nitshe, and A. PascualLeone, "A Sham-Controlled, Phase II Trial of TranscranialDirectCurrent Stimulation for the Treatment of Central Pain in Traumatic Spinal Cord Injury," Pain, vol.122, no,1-2, 2006, pp.197-209.

[19] C. Abbadie, and J.M. Besson, "c-fosExpression in Rat Lumbar Spinal Cord During the Development of Adjuvant-Induced Arthritis," Neuroscience, vol.48, no.4, 1992, pp.985-993.

[20] C.B. Hathaway, J.W. Hu, and D.A. Bereiter, "Distribution of Fos-Like Immunoreactivity in the Caudal Brainstem of the Rat Following Noxious Chemical Stimulation of the TempomandibularJoint," J Comp Neurol, vol.356, no.3, 1995, pp.444-456.

[21] J.A. Harris, "Using c-fos as a Neural Marker of Pain", Brain Res Bull, vol.45, no. 1, 1998, pp.1-8.

[22] S.J. Kim, "Mechanism of Motor Recovery Induced by Repeated Transcranial Direct Current Stimulation in Stroke Rat Model," Doctoral Dissertation, The Graduate School of Yonsei University, 2001.

[23] D.M. Basso, M.S. Beattie, and J.C. Bresnahan, "Graded Histological and LocomotorOutcomes after Spinal Cord Contusion Using the NYU Weight-Drop Device Versus Transection," ExpNeurol, vol.139, no.2, 1996, pp.224256.

[24] S.H. Kim, and J.M. Chung, "An Experimental Model for Peripheral Neuropathy Produced by Segmental Spinal Nerve Ligation in the Rat," Pain, vol.50, no.3, 1992, pp.355-363.

[25] S.R. Chaplan, A.B. Malmberg, and T.L. Yaksh, "Efficacy of Spinal NMDA Receptor Antagonism in Formalin Hyperalgesia and Nerve Injury Evoked Allodynia in the Rat," J PharmacolExpTher, vol.280, no.2, 1997, pp.829838.

[26] G. Paxinos, and C. Watson,TheRat Brain in Stereotaxic Coordinates. San Diego, Academic Press Inc., pp.13-38, 2005.

[27] B.D. Nicholson. "Evaluation and Treatment of Central Pain Syndromes," vol.62, no. 5, 2004, pp.S30-36.

[28] B.A. Wallace, K. Ashkan, and A.L. Benabid, "Deep Brain Stimulation for the Treatment of Chronic,
Intractable Pain," NeurosurgClin N Am, vol.15, no. 3, 2004, pp.343-357.

[29] A. Antal, M.A. Nitsche, W. Kruse, T.Z. Kincses, K.P. Hoffmann, and W. Paulus, "Direct Current Stimulation Over v5 Enhances VisuomotorCoordination by Improving Motion Perception in Humans," J CognNeurosci,vol.16, no.4, 2004, pp.521-527.

[30] S. Hesse, C. Werner, E.M. Schonhardt, A. Bardeleben, W. Jenrich, and S.G. Kirker, "Combined TranscranialDirectCurrent Stimulation and RobotAssisted Arm Training in SubacuteStrokePatients: APilotStudy," RestorNeurolNeurosci, vol.25, no.1, 2007, pp.9-15.

[31] A. Monti, F. Cogiamanian, S. Marceglia, R. Ferrucci, F. Mameli, S. Mrakic-Sposta, M. Vergari, S. Zago, and A. Priori, "Improved Naming after TranscranialDirectCurrent Stimulation in Aphasia," J NeurolNeurosurg Psychiatry, vol.79, no.4, 2008, pp.451453.

[32] A. Antal, N. Brepohl, C. Poreisz, K. Boros, G. Csifcsak, and W. Paulus, "TranscranialDirectCurrent Stimulation Over Somatosensory Cortex Decreases Experimentally Induced Acute Pain Perception," Clin J Pain, vol.24, no. 1, 2008, pp.56-63.

[33] P.S. Boggio, S. Zaghi, M. Lopes, and F. Fregni, "Modulatory Effects of Anodal TranscranialDirectCurrent Stimulation on Perception and Pain Thresholds in Healthy Volunteers," Eur J Neurol, vol.15, no. 10, 2008,pp.1124-1130.

[34] F. Fregni, R. Gimenes, A.C. Valle, M.J. Ferreira, R.R. Rocha, L. Natalle, R. Bravo, S.P. Rigonatti, S.D. Freedman, M.A. Nitsche, A. Pascual-Leone, and P.S. Boggio, "A Randomized, Sham-Controlled, Proof of Principle Study of TranscranialDirectCurrent Stimulation for the Treatment of Pain in Fibromyalgia,"Arthritis Rheum, vol.54, no. 12, 2006, pp.3988-3998.

[35] C.E. Hulsebosch, G.Y. Xu, J.R. Perez-Polo, K.N. Westlund, C.P. Taylor, and D.J. McAdoo, "Rodent Model of Chronic Central Pain after Spinal Cord Contusion Injury and Effects of Gabapentin," J Neurotrauma, vol.17, no. 12, 2000, pp.1205-1217.

[36] C.D. Mills, J.J. Grady, and C.E. Hulsebosch, "Changes in Exploratory Behavior as a Measure of Chronic Central Pain Following Spinal Cord Injury," J Neurotrauma, vol.18, no. 10, 2001, pp.1091-1105.

[37] D. Wachter, A. Wrede, W. Schulz-Schaeffer, A. Taghizadeh-Waghefi, M.A. Nitsche, A. Kutschenko, V. Rohde, and D. Liebetanz, "TranscranialDirectCurrent Stimulation Induces Polarity-Specific Changes of Cortical Blood Perfusion in the Rat," ExpNeurol, vol.227. no.2, 2011 pp.322-327.

[38] T. Kamida, S. Kong, N. Eshima, T. Abe, M. Fujiki, and H. Kobayashi, "TranscranialDirectCurrent Stimulation Decreases Convulsions and Spatial Memory Deficits Following Pilocarpine-Induced Status Epilepticus in Immature Rats," Behav Brain Res, vol.217, no.1, 2011, pp.99-103.

[39] Y.S. Gwak, T.S. Nam, K.S. Paik, C.E. Hulsebosch, and J.W. Leem, "Attenuation of Mechanical 
HyperalgesiaFollowingSpinal Cord Injury by Administration of Antibodies to Nerve Growth Factor in the Rat," NeurosciLett, vol.336, no. 2, 2003, pp.117-120.

[40] B.C. Hains, A.W. Everhart, S.D. Fullwood, and C.E. Hulsebosch, "Changes in Serotonin, Serotonin Transporter Expression and Serotonin Denervation Supersensitivity: Involvement in Chronic Central Pain after Spinal Hemisection in the Rat," ExpNeurol, vol.175, no. 2, 2002, pp.347-362.

[41] I. Omana-Zapata, M.A. Khabbaz, J.C. Hunter, and K.R.Bley, "QX-314 Inhibits Ectopic Nerve Activity Associated with Neuropathic Pain," Brain Res, vol.771, no. 2, 1997, pp.228-237.

[42] M.D. Christensen, A.W. Everhart, J.T. Pickelman, and C.E. Hulsebosch, "Mechanical and Thermal Allodynia in Chronic Central Pain Following Spinal Cord Injury," Pain, vol.68, no. 1, 1996, pp.97-107.

[43] R. Rolke, W. Magerl, K.A. Campbell, C. Schalber, S. Caspari, F. Birklein, and R.D. Treede, "Quantitative Sensory Testing: AComprehensiveProtocol for Clinical Trials," EurJ Pain, vol.10, no. 1, 2006, pp.77-88.

[44] C.G. Bachmann, R. Rolke, U. Scheidt, C. Stadelmann, M. Sommer, G. Pavlakovic, S. Happe, R.D. Treede,andW. Paulus, "Thermal Hypoesthesia Differentiates Secondary Restless Legs Syndrome Associated with Small FiberNeuropathy from Primary Restless Legs Syndrome," Brain, vol.133, no. 3, 2010, pp.762-770.

[45] E. Bullitt, "Expression of c-fos-Like Protein as a Marker for Neuronal Activity Following Noxious Stimulation in the Rat," J Comp Neurol, vol.296, no. 4, 1990, pp.517530.

[46] A.M. Strassman, and B.P. Vos, "Somatotopic and Laminar Organization of fos-Like Immunoreactivity in the Medullary and Upper Cervical Dorsal Horn Induced by Noxious Facial Stimulation in the Rat," J Comp Neurol, vol.331, no. 4, 1993, pp.495-516.

[47] M.A. Nitsche, A. Seeber, K. Frommann, C.C. Klein, C. Rochford, M.S. Nitsche, K. Fricke, D. Liebetanz, N. Lang, A. Antal, W. Paulus, and F. Tergau, "ModulatingParameters of Excitability During and after TranscranialDirectCurrent Stimulation of the Human Motor Cortex," J Physiol, vol.568, no. 1, 2005, pp.291303.

[48] T. Tsubokawa, Y. Katayama, T. Yamamoto, T. Hirayama, and S. Koyama, "Treatment of Thalamic Pain by Chronic Motor Cortex Stimulation," Pacing ClinElectrophysiol, vol.14, no.1,1991, pp.131-134.

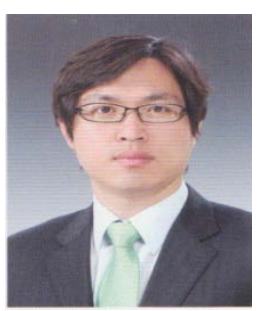

Kyung-Yoon Kim

He receivedthe doctoral degree in physical therapy from Dongshinuniversity, Korea in 2007. Now, he is a professor of physical therapy at Dongshin university of Korea. His main research interests include therapeutic exercise and neuroscience.

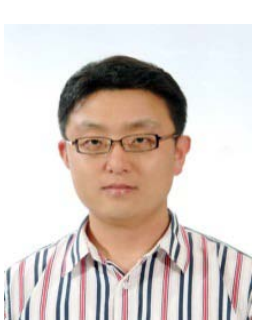

\section{Ki-CholSim}

He received the M.S., Ph.D. in physical therapy science from Dongshinuniversity, Korea in 2011. His main research interests include neuroscience and neurophysiology.

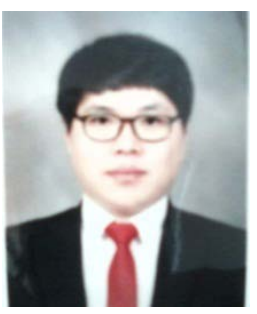

\section{Hyun-Seung Kim}

He receivedthe bachelor degree in physical therapy from Seonamuniversity, Korea in 2005. Now, he attend to a master's degree program in physical therapy from Sahmyook university. $\mathrm{He}$ works for Uijeongbu healings hospital. His main research interests include neurological physical therapy and therapeutic exercise.

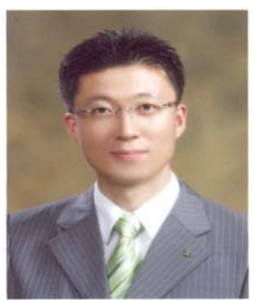

\section{Wan-Suk Choi}

He receivedtheM.S., Ph.D. in physical therapyscience from Yonginuniversity, Korea in 2009. Since 2010, he has been with International University of Korea. His main research interests include therapeutic exercise and manual therapy.

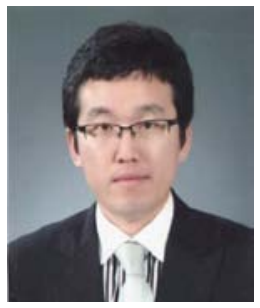

\section{Gi-Do Kim}

He received the M.S., Ph.D. in physicaltherapy science from Dongshinuniversity,Korea in 2010. Since 2011, he has been with International University of Korea. His main research interests include neuroscience and neurophysiology 